Correcting for electrostatic cutoffs in free energy simulations: Toward consistency between simulations with different cutoffs

Haluk ResatJ. Andrew McCammon

Citation: The Journal of Chemical Physics 108, 9617 (1998); doi: 10.1063/1.476437

View online: http://dx.doi.org/10.1063/1.476437

View Table of Contents: http://aip.scitation.org/toc/jcp/108/23

Published by the American Institute of Physics

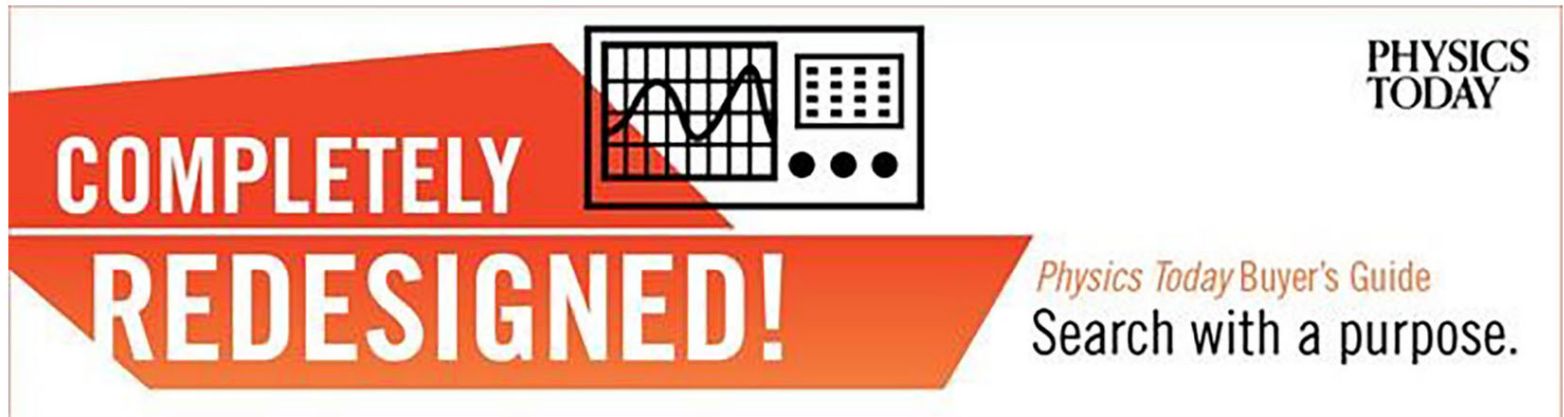




\title{
Correcting for electrostatic cutoffs in free energy simulations: Toward consistency between simulations with different cutoffs
}

\author{
Haluk Resat \\ Department of Physics, Faculty of Arts and Sciences, Koc University, Istinye, Istanbul, Turkey \\ J. Andrew McCammon \\ Department of Chemistry and Biochemistry and Department of Pharmacology, \\ University of California at San Diego, La Jolla, California 92093-0365
}

(Received 7 October 1997; accepted 10 March 1998)

\begin{abstract}
The use of electrostatic cutoffs in calculations of free energy differences by molecular simulations introduces errors. Even though both solute-solvent and solvent-solvent cutoffs are known to create discrepancies, past efforts have mostly been directed toward correcting for the solute-solvent cutoffs. In this work, an approach based on the generalized reaction field formalism is developed to correct for the solvent-solvent cutoff errors as well. It is shown using a series of simulations that when the cutoff lengths are significantly smaller than the half unit cell size, and the solute-solvent cutoff is not much larger than the solvent-solvent cutoff, the new algorithm is able to yield better agreement among simulations employing different truncation lengths. () 1998 American Institute of Physics. [S0021-9606(98)50723-4]
\end{abstract}

\section{INTRODUCTION}

One of the challenges of molecular simulations has been the accurate calculation of free energies. Even though the idea and basic theory behind such calculations are straightforward, their numerical results prove to be algorithm dependent. ${ }^{1,2}$ In particular, handling of the long-ranged electrostatic interactions requires special care. This is reflected in the quantitatively different results obtained when different periodic boundary conditions are used, or when various cutoffs are handled differently., ${ }^{3,4}$ There have been attempts to correct for the approximations employed. For example, methods based on the Born formula, ${ }^{5}$ on the generalized reaction field formula, ${ }^{6-9}$ and recently on the numerical solution of the Poisson equation, ${ }^{10}$ have been advocated to approximately account for the truncation of the solute-solvent interactions. Similar approaches have been suggested to eliminate the finite size effects due to the use of periodic boundary conditions. ${ }^{1-14}$ A particular challenge, however, seems to arise due to the truncation of the solvent-solvent interactions. ${ }^{3,4}$ Work of Straatsma and Berendsen ${ }^{3}$ clearly showed that different spherical solvent-solvent truncation radii give different free energies. Wood's group ${ }^{4,14}$ later developed a method to successfully estimate the solventsolvent truncation errors for single solute atom cases.

An alternative to the use of truncations is the use of Ewald sum techniques. ${ }^{15}$ Even though they have been shown to be successful and provide consistent results, ${ }^{11-13}$ simulations using the Ewald sum approach correspond to a concentrated solution rather than the infinite dilution that is sometimes desired. For example, a free energy molecular simulation of an ion with 1000 waters would correspond to 55 millimolar concentration. To further lower the concentration to micromolar range, large simulation cell sizes are needed which increases the computational cost beyond reasonable limits. Another limitation of the Ewald sum tech- nique arises when the total charge of the system is nonzero. In that case, one generally adds a uniform neutralizing background charge distribution. Even though there are proposed ways to correct for this modification, ${ }^{11-13}$ this alters the system Hamiltonian, and probably, the physical properties of the system.

In this work, we build on an earlier study, ${ }^{10}$ and develop an algorithm based on the generalized reaction field formalism to eliminate the solvent-solvent cutoff and periodicity effects from the simulations. That is, the new algorithm allows us to extrapolate the simulation results with cutoffs and periodic boundary conditions to the infinite dilution limit. We show with numerical examples that, when the cutoff lengths are relatively smaller than the half unit cell size, the algorithm makes it possible to achieve consistent results between simulations employing different solute-solvent and solvent-solvent spherical cutoff lengths.

\section{THEORY}

\section{A. Background}

In free energy simulations, one estimates the ensemble average of a functional of the Hamiltonian with respect to the Boltzmann factors based on the full Hamiltonian. For example, in the thermodynamic integration method, ${ }^{1,2,16}$ the free energy difference between the initial and final states is given as

$$
\Delta G=\int_{i}^{f}\left\langle\frac{\partial \mathscr{H}(\lambda)}{\partial \lambda}\right\rangle_{\mathscr{H}} d \lambda,
$$

where $\lambda$ is the coupling parameter and $\langle\cdots\rangle_{\mathscr{H}}$ implies a Boltzmann average based on the Hamiltonian $\mathscr{H}(\lambda)$. In molecular simulations employing truncations, certain molecules or groups do not get affected by the forces due to other molecules/groups which are not within the defined interac- 
tion volume ${ }^{15}$ Such truncations introduce approximations. In other words, it is assumed in calculating the ensemble averages that

$$
\langle\cdots\rangle_{\mathscr{H}} \approx\langle\cdots\rangle_{\mathscr{G} *},
$$

where $\mathscr{H}^{*}$ is the truncated Hamiltonian. Since parts of the Hamiltonian are neglected, this approximation implicitly introduces errors in the quantitative results. Existing correction methods try to include contributions due to the neglected interactions

$$
\Delta G_{\text {corr }}=\Delta G(\text { with } \mathscr{H})-\Delta G\left(\text { with } \mathscr{H}^{*}\right),
$$

in a somewhat empirical manner. If both solute-solvent and solvent-solvent truncations are employed, $\Delta G_{\text {corr }}$ can be separated into two contributions, $\Delta G_{\text {corr }}(I)$ and $\Delta G_{\text {corr }}(W)$, due to the solute-solvent and the solvent-solvent cutoffs, respectively.

For example, let us assume that a simulation involves an ion whose charge changes from $Q_{i}$ to $Q_{f}$ and that its interaction with the solvent molecules is modeled using a spherical cutoff of radius $r=R_{c}$. In terms of the forces exerted on the solute molecule, this model is equivalent to rigidly placing the solute and the solvent molecules lying within a sphere of radius $R_{c}$ from the solute group into a vacuum (rather than a dense dielectric solution) and then calculating the forces on the solute. In this case, the error in the solvation free energy due to the solute-solvent truncation error can be estimated to a good degree using a simple Born correction ${ }^{5}$

$$
\Delta G_{\text {corr }}(I)=-\frac{1}{2}\left(\frac{1}{\epsilon_{\text {in }}}-\frac{1}{\epsilon_{s}}\right) \frac{Q_{f}^{2}-Q_{i}^{2}}{R_{c}},
$$

where $\epsilon_{s}$ and $\epsilon_{\text {in }}$, respectively, are the dielectric constants of the surrounding dielectric medium and of the interior of the cavity.

In generalizing Eq. (4), the free energy of a distribution of $N$ point charges $\{q\}$ inside a cavity of radius $R_{c}$ in a dielectric continuum is given by the generalized reaction field (GRF) formula $3,6,10$

$$
G=-\frac{1}{2 \epsilon_{\text {in }}} \sum_{n=0}^{\infty} \frac{(n+1)\left(\epsilon_{r}-1\right)}{(n+1) \epsilon_{r}+n} \frac{Q_{n}}{R_{c}^{2 n+1},}
$$

where $\epsilon_{r} \equiv \epsilon_{s} / \epsilon_{\text {in }}$, and $Q_{n}$ is the square of the $n$th moment of the charge distribution

$$
Q_{n}=\sum_{k=1}^{N} \sum_{l=1}^{N} q_{k} q_{l} r_{k}^{n} r_{l}^{n} P_{n}\left(\cos \theta_{k l}\right) .
$$

In Eq. (5b) $P_{n}(\cos \theta)$ is the Legendre polynomial of order $n$, and $r_{k}$ is the distance from the center of the cavity of the $k$ th site with charge $q_{k}$. Note that the Born formula, Eq. (4), follows from the single site limiting case of Eqs. (5) for $n$ $=0$. In applying the above formula, solvent molecules within the solute-solvent interaction cutoff sphere would form some of the $N$ sites within the cavity to be treated with the GRF approach. If one's aim is to correct only for the solutesolvent electrostatic interaction cutoff effects, one of the sums in Eq. (5b) should be done over the solute sites only. ${ }^{10}$

The above formulas are based on the continuum dielectric solution of the Poisson equation for simple geometries.
We have recently advocated the use of numerical solutions of the Poisson (or the Poisson-Boltzmann) equation which has the advantage that it avoids the restriction to simpler geometries with a reasonable increase in the computational expense. ${ }^{10}$

All of the above approaches, however, are mainly concerned with solute-solvent truncation errors only. Works by Straatsma and Berendsen ${ }^{3}$ and by Wood ${ }^{4}$ showed that correcting for the solute-solvent truncation errors only does not allow one to achieve consistent results among various simulations differing in the boundary conditions or in the spherical solvent-solvent truncation radius. Careful handling of the solvent-solvent interactions seem to be as important as the solute-solvent interactions. ${ }^{4}$

The numerical method for using the Poisson equation to correct for truncation errors, developed in Ref. 10 can be easily generalized to treat the effects due to the truncation of the interaction of solvent molecules. This would, however, require solving the Poisson equation for each water molecule in the system. If one analyzes 100 sample configurations, and if there are 1000 waters, this would require the iterative solution of the Poisson equation $10^{5}$ times. Even though this would give an accurate estimation of the cutoff errors, this approach certainly would not be economical. Therefore, simpler and quantitatively reliable analytical approaches should be favored. A hint that the performance of simpler analytical approaches, such as the GRF, are reasonable for simple geometries was observed in our earlier study. ${ }^{10}$ In this work, we present an algorithm based on the GRF which allows us to eliminate the solvent cutoff errors from the free energy simulation results for a periodic system.

\section{B. Elimination of the cutoff errors}

In a molecular free energy simulation such as thermodynamic integration, Eq. (1), using periodic boundary conditions with the minimum image convention and truncations of interactions, one actually calculates the free energies for a periodic system with an approximate Hamiltonian. To obtain the free energies at infinite dilution, the effects of periodicity, i.e., the finite size effects, and the effects of the truncations need to be corrected. For this, the analytical generalized reaction field (GRF) method may prove to be useful. Implementation of the GRF to correct for the solvent cutoff errors closely follows that of the solute-solvent cutoff correction presented in Ref. 10. In this approach, one forms an interaction sphere for each solvent molecule and calculates the corresponding reaction field correction

$$
G=\frac{1}{2} \sum_{k} q_{k} \Phi_{k},
$$

where the $k$ summation is over the interaction sites of the central solvent molecule, and $\Phi_{k}$ is the reaction field potential at site $k$ due to all the charges (including solute molecule charges if the solute group is inside) enclosed in the cavity. ${ }^{10}$ If the radius of the spherical cavity is $R_{c}$, for $\epsilon_{\mathrm{in}}=1$, the reaction field potential $\Phi_{k}$ is given by Eq. (A3) of the Appendix ${ }^{17}$ 


$$
\Phi_{k}=\sum_{n=0}^{\infty} \frac{(n+1)\left(1-\epsilon_{r}\right)}{(n+1) \epsilon_{r}+n} \frac{r_{k}^{n}}{R_{c}^{2 n+1}} \sum_{l=1} q_{l} r_{l}^{n} P_{n}\left(\cos \theta_{k l}\right) .
$$

Here, the $l$ summation is over all the charge sites within the cavity. Correction $G$ is calculated separately for each solvent molecule in the simulation unit cell and summed.

However, a slight complication arises when the charged solute atom (or some solute groups) are outside the interaction sphere of the solvent molecule. To have a complete picture, such polarization effects should be included as well. The problem of finding the electrostatic potential distribution when a point charge resides outside a dielectric sphere is analytically solvable using a continuum dielectric reaction field approach (solution is given in the Appendix)

$$
\Phi_{k}=\sum_{n=0}^{\infty} \frac{(n+1)\left(1-\epsilon_{r}\right)}{(n+1) \epsilon_{r}+n} r_{k}^{n} \sum_{l=1}^{N_{\text {out }}} \frac{q_{l}}{r_{l}^{n+1}} P_{n}\left(\cos \theta_{k l}\right),
$$

where $\epsilon_{\text {in }}$ is set equal to 1 . Summation $l$ is over the charged sites of the solute group which are outside the solvent molecule's interaction sphere.

The overall correction to the free energy due to the reaction field of the solute ion when the solute is outside the interaction sphere of the solvent molecule, i.e., the above case, can be calculated analytically if one assumes a homogeneous solvent distribution, and an orientational ordering described by a mean field. Using the analytical solution, this contribution would be (details are given in the Appendix)

$$
G=\frac{4 \pi \rho_{w}}{3}\left(\frac{\epsilon_{r}-1}{2 \epsilon_{r}+1}\right)\left(\frac{q^{2} \mu^{2}}{k_{\mathrm{B}} T \epsilon_{s}}\right)\left(\frac{1}{R_{c}}\right) .
$$

In the above equation $\rho_{w}=N / V$ is the water number density, $\mu$ is the dipole moment of a water molecule, $T$ is temperature, and $k_{\mathrm{B}}$ is the Boltzmann constant.

In the calculation of the cutoff errors due to the truncation of the interaction of a solvent molecule with the others, there can be difficulties when the charged ion solute crosses the boundary of the solvent interaction sphere. Crossing of a charged particle through the dielectric boundary alters the induced charge distribution at the boundary and gives rise to discrepancies. If this electrostatic problem is solved using the image charges method, ${ }^{18}$ this gets reflected as an image charge placed at the boundary between the two dielectric media. The above equations also show that, if one looks to the $n=1$ term, the reaction field potential inside the low dielectric sphere is different when the solute ion is slightly inside or outside the dielectric boundary. However, this discrepancy is due to the idealized solution of the problem; in nature, through penetration of the low dielectric regions of the solute and the solvent interaction sphere, such irregularities would be smoothed out.

The above equations would give an estimate of the cutoff correction due to the truncation of the interaction of the solvent molecules. Note that these equations miss two effects which might be important. First, since the analyzed configurations are obtained from a molecular simulation employing periodic boundary conditions, the above summations can only be done for solvent molecules inside the unit cell. This is equivalent to the assumption that the solvent contribution to the reaction field properties of waters at large separations from the solute molecule are the same for both transformation end states of the solute molecule. Interactions at further distances are assumed to cancel. If the screening effects of the solvent are not sufficient, or when there are "surface" irregularities due to small unit cells, this assumption may not hold. The latter problems arise particularly when the cutoff distances are comparable to the half unit cell size. Note that if this assumption is valid, the free energies calculated correspond to the free energies at the infinite dilution limit. This is because of the fact that, for each molecule in the system, the correction scheme extends their interaction volumes to infinity as if it is an inhomogeneous nonperiodic system. As reported in the results sections, for two of the performed simulations, this assumption seems to break down when the solute-solvent cutoff radius is much larger than the solventsolvent cutoff radius and is closer to the half unit cell size.

The second effect reflects that the continuum dielectric treatment assumes that the reaction field is created by a point charge imbedded into the dielectric continuum. These charges actually occupy a finite space and the dielectric constant would be different around these charges. It was shown by Davis that such finite size effects can be important. ${ }^{19}$ Davis extensively investigated overlapping dielectric medium effects and presented ways to incorporate them into the calculations for simple geometries. ${ }^{19}$ Unfortunately, it is still not easy to include similar corrections when the polar groups have nonideal geometries. Therefore, inclusion of the finite size effects will be postponed to a future study.

\section{CALCULATIONS}

The calculations closely follow those of Ref. 10. First, a free energy calculation is performed to obtain the free energy difference with the employed truncations, Eq. (1). To implement the above cutoff correction algorithm, one then generates sample molecular simulation configurations for both end states, i.e., the initial and the final states between which the free energy difference is being determined. Those configurations are later used in Eq. (6) to calculate the required correction for both states separately. Subtracting the calculated correction for the initial state from the one for the final state would give the overall solvent truncation cutoff correction to the $\Delta G$ of the studied transition.

\section{A. Thermodynamic integration free energy simulations}

In this work, we have studied the transformation of a neon atom into a sodium cation in aqueous solution. Simulations were done using the ARGOS molecular dynamics program. ${ }^{20}$ The transformation coordinate was covered in 21 steps with uniform intervals. Force field parameters developed by Soman and Straatsma ${ }^{21}$ were used: $\sigma_{\mathrm{Na}}=3.616 \AA$, $\sigma_{\mathrm{Ne}}=3.036 \AA, \epsilon_{\mathrm{Na}}=0.00169 \mathrm{kcal} / \mathrm{mol}$, and $\epsilon_{\mathrm{Ne}}=0.03689$ $\mathrm{kcal} / \mathrm{mol}$. There were 1428 water molecules in the cubic unit cell, and the SPC/E model ${ }^{22}$ was used to characterize the water. Calculations with periodic boundary conditions were performed in the constant $(N, p, T)$ ensemble with temperature and pressure coupling at $298.15 \mathrm{~K}$ and $1 \mathrm{~atm}$. This, on 
average, corresponded to an unit cell box sidelength of 34.9 $\AA$. The solvent molecular geometries were kept rigid using the SHAKE algorithm. ${ }^{23}$ Intermolecular $\sigma$ 's and $\epsilon$ 's were determined by utilizing a geometric mixing rule. Simulations at each thermodynamic integration point $\lambda_{i}$ were run for 3000 steps with a time step of $2 \mathrm{fs}$ following an equilibration of 500 steps. Each $\lambda_{i}$ run was started using the final configuration of the previous transformation coordinate $\lambda_{i-1}$ run. Solute-solvent and solvent-solvent interactions were truncated using spherical cutoffs.

To investigate whether consistency can be achieved when various truncation radii are used, a series of simulations differing in the solute-solvent and solvent-solvent electrostatic cutoff lengths were performed. A total of six simulations were run which can be grouped into two categories: In the first group, the electrostatic solvent-solvent truncation radius was $9 \AA$, and the solute-solvent cutoff radius was varied as $9,11,13$, and $15 \AA$. In the second group, the solute-solvent cutoff radius was set at $11 \AA$, and the solvent-solvent radius was changed from 9 to $11 \AA$, and to $13 \AA$. In all simulations, the cutoff length for the short range potentials, i.e., the Lennard-Jones interaction terms, was $9 \AA$ for both the solute-solvent and the solvent-solvent interactions.

\section{B. Solute cutoff correction}

This contribution, $\Delta G_{\text {corr }}(I)$, is calculated as in Ref. 10. To briefly summarize, the Poisson equation was solved for the sample configurations collected during molecular dynamics simulations at the end points. 200 sample configurations for each simulation were used in the analysis. The Poisson equation calculations were done with the UHBD program package ${ }^{24}$ using a finite difference algorithm with focusing to a final grid spacing of $0.1 \AA$. A radius of $1.2 \AA$ was used for the hydrogen sites to define the volumes occupied by the water hydrogens. The solute and the solvent molecules within the MD cutoff radius were placed into a dielectric continuum having a dielectric constant of 78. A dielectric constant of 1 was assigned to the interior of the solutesolvent solvation complex. Further details may be found in Ref. 10.

\section{Solvent cutoff correction}

As in the solute cutoff case, configurations collected during the molecular dynamics simulations at the end points were used. Depending on the convergence characteristics of the simulations, between 200 and 500 configurations were analyzed for each simulation. To compare various contributions to the solvent cutoff corrections, the contribution of the solvent molecules and of the solute were treated separately. For this, the reaction field $\Phi_{k}$ in Eq. (6) was separated into two components; one due to the reaction field of the enclosed solvent molecules, and a second one due to the effects of the charged solute

$$
\begin{aligned}
G_{\text {corr }}(W) & =\frac{1}{2} \sum_{k} q_{k} \Phi_{k, i}+\frac{1}{2} \sum_{k} q_{k} \Phi_{k, w} \\
& \equiv G_{\text {corr }, w i}(W)+G_{\text {corr } w w}(W) .
\end{aligned}
$$

TABLE I. Thermodynamic integration free energy and solute cutoff correction results. ${ }^{\text {a }}$

\begin{tabular}{rrrrc}
\hline \hline$R_{i, s}$ & $R_{s, s}$ & $\Delta G(T I)$ & $\Delta G_{\text {corr }}(I)$ & $\Delta G(T I+I)$ \\
\hline 9 & 9 & -87.36 & -15.38 & -102.74 \\
11 & 9 & -94.27 & -12.37 & -106.64 \\
13 & 9 & -99.63 & -10.23 & -109.86 \\
15 & 9 & -104.54 & -8.92 & -113.46 \\
11 & 9 & -94.27 & -12.37 & -106.64 \\
11 & 11 & -88.08 & -12.43 & -100.51 \\
11 & 13 & -86.56 & -12.58 & -99.14 \\
\hline \hline
\end{tabular}

${ }^{\mathrm{a}} R_{i, s}$ and $R_{s, s}$ are the solute-solvent and the solvent-solvent cutoff radius, respectively. Free energy results are in $\mathrm{kcal} / \mathrm{mol} . \Delta G_{\text {corr }}(I)$ in the solutesolvent cutoff correction calculated using the Poisson equation (Ref. 10).

The reaction field of the solvent molecules, $\Phi_{k, w}$, was calculated using Eq. (7a). The solute contribution, $\Phi_{k, i}$, when the ion was inside the reaction field sphere was determined using the same equation. Solute contribution to the free energy when the ion was outside the reaction field sphere was included using the analytical result given by the Eq. (8).

In the GRF calculations, a somewhat arbitrary average cutoff radius, $R_{c}$, has to be defined. For this, we adapt Wang and Hermans' approach ${ }^{8}$ and define $R_{c}$ to be equal to the MD truncation distance plus an allowance of $1.6 \AA$ for the water molecules residing close to the surface of the complex. A value of 78 was assigned to the $\epsilon_{r}$. In implementing Eq. (7a), it was observed that the series converges rapidly. Therefore, only the first four terms of the series were included in the calculations.

\section{RESULTS}

Table I summarizes the thermodynamic integration free energy, $\Delta G(T I)$, and the solute cutoff correction, $\Delta G_{\text {corr }}(I)$, results. These results, in accordance with earlier studies, ${ }^{3,4}$ clearly show that calculated free energies depend on the cutoff radius quite strongly. Adding the correction factor due to the solute-solvent cutoffs, $\Delta G(T I+I)$, only marginally improves the agreement between the results.

As discussed earlier, to obtain accurate free energy results, one has to correct for the solvent cutoff effects as well. One such approach based on the generalized reaction field formalism was developed in Sec. II B. Results calculated using this method, $\Delta G(T I+I+W)$, are tabulated in Table II.

TABLE II. Free energy results at infinite dilution limit with solvent cutoff correction. $^{\mathrm{a}}$

\begin{tabular}{rrrccc}
\hline \hline$R_{i, s}$ & $R_{s, s}$ & $\Delta G(T I+I)$ & $\Delta G_{\text {corr }, w w}(W)$ & $\Delta G_{\text {corr }, w i}(W)$ & $\Delta G(T I+I+W)$ \\
\hline 9 & 9 & -102.74 & -2.52 & 11.06 & -94.20 \\
11 & 9 & -106.64 & -1.64 & 14.71 & -93.57 \\
13 & 9 & -109.86 & -7.94 & 14.86 & -102.94 \\
15 & 9 & -113.46 & -9.42 & 15.09 & -107.79 \\
11 & 9 & -106.64 & -1.64 & 14.71 & -93.57 \\
11 & 11 & -100.51 & -2.24 & 9.56 & -93.19 \\
11 & 13 & -99.14 & -1.28 & 6.85 & -93.57 \\
\hline \hline
\end{tabular}

${ }^{\mathrm{a}} R_{i, s}$ and $R_{s, s}$ are the solute-solvent and the solvent-solvent cutoff radius, respectively. $\Delta G_{\text {corr }}(W)$ is the solvent cutoff correction calculated using the GRF formulas. Free energy results are in $\mathrm{kcal} / \mathrm{mol}$. 
Estimated overall error bars in the reported free energy values are about $2 \mathrm{kcal} / \mathrm{mol}$. There is good agreement among four of the six simulations. However, the agreement of these four simulations with the results of simulations in which the difference of the cutoff radius $R_{i, s}-R_{s, s}$ is large is rather unsatisfactory. There could be two possible main sources of this disagreement: (i) Different cutoffs would give rise to different "surface" effects. Such effects (which may combine with the possible finite unit cell size effects) would make the simulated systems genuinely different from each other. Therefore, if the physical systems are different, extrapolation of the free energies to the infinite dilution limit should actually give different results. (ii) Because of the simplistic picture employed, such as point charges immersed in dielectric continuum, the generalized reaction field approach itself incurs some error.

For the simulations reported in this work, the discrepancy is probably due to a combination of both factors. As pointed out by Wood, ${ }^{4}$ when $R_{s, s}$ is less than $R_{i, s}$, the solvent cutoff corrections and the periodicity effects can be as large as the solute cutoff corrections. Because of the large negative $\Delta G_{\text {corr } w w}(W)$ values, our results for large $R_{i, s}$ $-R_{s, s}$, however, give a solvent cutoff correction which is rather sizable. This might be due to the inadequacy of the reaction field method for such cases. When $R_{i, s}-R_{s, s}$ is large, GRF includes the interaction between solvent molecules close to the surface of the solute interaction sphere and solvent molecules next to the solute in a dielectric continuum way. Since waters next to the solute may be highly ordered such continuum representations may not be adequate.

Truncation of the intermolecular interaction potentials is known to introduce unphysical distribution properties. For example, the pair distribution functions and electrostatic potential distributions do not decay to their large $r$ limiting values smoothly; there may be dips or bumps in the distribution functions. As shown by Ashbaugh and Wood $^{14}$ the contribution to the electrostatic potential at the central ion site due to molecule-based cutoffs can be as large as 19 $\mathrm{kcal} / \mathrm{mol}$ when $9 \AA$ spherical cutoff lengths are used. Although this contribution will depend on the cutoff length etc, this result can be taken as an indicator of the magnitude of errors involved.

The unit cell half-boxlength was about $17.5 \AA$ in the simulations. When the half-boxlength is not much larger than the cutoff radius, the unit cell boundary effects can become important. As stated above as a possible source of error, use of solute-solvent cutoff lengths $R_{i, s} \geqslant 13 \AA$ might have changed the Hamiltonian such that the extrapolation to the infinitely dilute solution limit corresponds to a different physical system. Thus there actually should be differences in the results which might be what is observed. When the methods described in this work are used, therefore, it is best to use cutoff lengths that are significantly smaller than the halfboxlength.

\section{SUMMARY}

In this work, an approach based on the generalized reaction field formalism was developed for the elimination of the solvent cutoff effects from the molecular simulations. As discussed in Sec. II B, when the surface irregularities are expected to be unimportant, the algorithm developed here also allows the extrapolation of the free energy results to the infinitely dilute solution limit. The applicability and performance of the algorithm was measured in a series of simulations to calculate the solvation free energy difference between a neon atom and a sodium cation in aqueous solution. Series of simulations employing different solutesolvent and solvent-solvent truncation lengths were performed. Comparison of the results showed that the cutoff correction algorithm works satisfactorily well when the solvent-solvent cutoff radius $R_{s, s}$ is larger than the solutesolvent cutoff radius $R_{i, s}$, or when the solute-solvent cutoff radius is slightly larger than the solvent-solvent cutoff radius. When $R_{i, s}-R_{s, s}$ becomes large and $R_{i, s}$ is close to the halfbox size, the GRF approach seems to start breaking down. However, such deficiencies might be improved by including the recent developments in finite size dielectric representation of solutes. ${ }^{19}$

As a final note, it has to be pointed out that the algorithm developed here has the drawbacks which are characteristics of the generalized reaction field approach. For example, it is applicable only to idealized geometries such as spherical or ellipsoidal shapes; crossing of the dielectric boundary by a charged particle cannot be properly represented; and there are ambiguities in defining the cavity with different dielectric constant. Because of these problems, development of different cutoff correction schemes which can address some of the above shortcomings could still be quite beneficial.

\section{ACKNOWLEDGMENTS}

This work was supported in part by grants to J.A.M. from NSF, NIH, and SDSC.

\section{APPENDIX}

\section{Finding the reaction potential inside a spherical cavity due to a point charge placed inside the sphere}

The solution to this problem is also known as the Kirkwood formula. ${ }^{6}$ In this case, the electrostatic potentials inside and outside the sphere are given by an expansion in terms of the Legendre polynomials $P_{n}(\cos \theta)$. Let us consider a spherical cavity of radius $R_{c}$ with an interior dielectric constant of $\epsilon_{\text {in }}$ immersed in a medium of dielectric constant $\epsilon_{s}$. To utilize the symmetry of the system, one defines the $z$ axis as the axis passing through sphere's center and the ion's location and $\theta$ as the angle with respect to this $z$ axis. If the ion has a charge of $q$, and $\tau$ donates the distance of the ion from the sphere's center, then ${ }^{25}$ 


$$
\begin{aligned}
\Phi_{\text {in }}(r, \theta)= & \sum_{n=0}^{\infty} A_{n} r^{n} P_{n}(\cos \theta) \\
& +\frac{q}{\epsilon_{\text {in }}} \sum_{n=0}^{\infty} \frac{r^{n}}{\tau^{n+1}} P_{n}(\cos \theta) \quad \text { if } r<\tau, \\
\Phi_{\text {in }}(r, \theta)= & \sum_{n=0}^{\infty} A_{n} r^{n} P_{n}(\cos \theta) \\
& +\frac{q}{\epsilon_{\text {in }}} \sum_{n=0}^{\infty} \frac{\tau^{n}}{r^{n+1}} P_{n}(\cos \theta) \quad \text { if } r>\tau,
\end{aligned}
$$

and

$$
\Phi_{\text {out }}(r, \theta)=\sum_{n=0}^{\infty} \frac{B_{n}}{r^{n+1}} P_{n}(\cos \theta) .
$$

The electrostatic field should satisfy the boundary conditions: (i) tangential component of the electric field is continuous, i.e., $\Phi_{\text {in }}(r, \theta)=\Phi_{\text {out }}(r, \theta)$, and (ii) normal component of the displacement field is continuous, i.e., $\epsilon_{\text {in }}\left[\partial \Phi_{\text {in }}(r, \theta) / \partial r\right]$ $=\epsilon_{s}\left[\partial \Phi_{\text {out }}(r, \theta) / \partial r\right]$ at $r=R_{c}$. Using the orthogonality of the Legendre polynomials, these boundary conditions can be used to solve for the unknown $A_{n}$ and $B_{n}$ coefficients. They are given by

$$
B_{n}=\frac{q}{\epsilon_{\mathrm{in}}} \frac{(2 n+1) \tau^{n}}{n+\epsilon_{r}(n+1)}
$$

and

$$
A_{n}=\frac{q}{\epsilon_{\mathrm{in}}} \frac{(n+1)\left(1-\epsilon_{r}\right)}{n+\epsilon_{r}(n+1)} \frac{\tau^{n}}{R_{c}^{2 n+1}}
$$

where $\epsilon_{r} \equiv \epsilon_{s} / \epsilon_{\text {in }}$. From Eqs. (A2), the reaction field potential inside the cavity can be calculated

$$
\begin{aligned}
\Phi_{\text {r.f. }} & \equiv \Phi_{\text {in }}-\Phi_{\text {in }}\left(\epsilon_{s}=\epsilon_{\text {in }}\right) \\
& =\frac{q}{\epsilon_{\text {in }}} \sum_{n=0}^{\infty}\left(\frac{(n+1)\left(1-\epsilon_{r}\right)}{n+\epsilon_{r}(n+1)}\right) \frac{\tau^{n} r^{n}}{R_{c}^{2 n+1}} P_{n}(\cos \theta) .
\end{aligned}
$$

\section{Finding the reaction potential inside a spherical cavity due to a point charge placed outside the sphere}

The solution to this problem follows closely that of the previous section. Using the same geometrical description, the electrostatic potentials are given by the following formulas:

$$
\begin{aligned}
\Phi_{\text {in }}(r, \theta)= & \sum_{n=0}^{\infty} A_{n} r^{n} P_{n}(\cos \theta) \\
\Phi_{\text {out }}(r, \theta)= & \sum_{n=0}^{\infty} \frac{B_{n}}{r^{n+1}} P_{n}(\cos \theta) \\
& +\frac{q}{\epsilon_{s}} \sum_{n=0}^{\infty} \frac{r^{n}}{\tau^{n+1}} P_{n}(\cos \theta) \quad \text { if } r<\tau
\end{aligned}
$$

and

$$
\begin{aligned}
\Phi_{\text {out }}(r, \theta)= & \sum_{n=0}^{\infty} \frac{B_{n}}{r^{n+1}} P_{n}(\cos \theta) \\
& +\frac{q}{\epsilon_{s}} \sum_{n=0}^{\infty} \frac{\tau^{n}}{r^{n+1}} P_{n}(\cos \theta) \quad \text { if } r>\tau .
\end{aligned}
$$

Again using the boundary conditions, unknown $A_{n}$ and $B_{n}$ coefficients can be determined ${ }^{19}$

$$
B_{n}=\frac{q}{\epsilon_{s}} \frac{n\left(\epsilon_{r}-1\right)}{n+\epsilon_{r}(n+1)} \frac{R_{c}^{2 n+1}}{\tau^{n+1}}
$$

and

$$
A_{n}=\frac{q}{\epsilon_{\mathrm{in}}} \frac{(2 n+1)}{n+\epsilon_{r}(n+1)} \frac{1}{\tau^{n+1}} .
$$

Thus the reaction field potential inside the cavity is

$$
\Phi_{\text {r.f. }}=\frac{q}{\epsilon_{\text {in }}} \sum_{n=0}^{\infty}\left(\frac{(n+1)\left(1-\epsilon_{r}\right)}{n+\epsilon_{r}(n+1)}\right) \frac{r^{n}}{\tau^{n+1}} P_{n}(\cos \theta) .
$$

\section{Calculating the solvent cutoff correction due to the solute contribution when the solute is outside the interaction sphere}

Given a homogeneous solvent assumption, and an orientational ordering described by a mean field, the effects of the solute on the solvent cutoffs when the ion is outside solvent's interaction sphere can be analytically calculated. For such cases, the solvent for which the cutoff correction is being calculated can be represented by a point dipole $\mu$ located at the origin (fine details due to the molecular structure of waters would be unimportant when the distance between molecules are large). Then one has to calculate the correction to the free energy using the reaction field given by Eq. (A6) for the $n=1$ term only. In this limit the contribution to the free energy $G=q \Phi / 2$ would be

$$
G(\tau, \theta)=\frac{q}{\epsilon_{\text {in }}}\left(\frac{1-\epsilon_{r}}{1+2 \epsilon_{r}}\right) \frac{\mu}{\tau^{2}} P_{1}(\cos \theta) .
$$

One has to include the contribution for all possible ion locations, i.e., an integral of this reaction field over the volume available to the solute would give the total cutoff correction

$$
G=2 \pi \int_{\tau=R}^{\infty} d \tau \tau^{2} \int_{-1}^{1} d(\cos \theta) G(\tau, \theta) P_{\text {ion }}(\tau, \theta),
$$

where $P_{\text {ion }}$ is the probability of the ion to be at the position $(\tau, \theta)$. Using a mean field type approach this probability can be factored as $P_{\text {ion }}(\tau, \theta)=(1 / V) C(\tau) P^{*}(\tau, \theta)$. The angle dependent part can be described by the Boltzmann factor $P^{*}(\tau, \theta)=\exp \left(-\beta q \mu \cos \theta / \epsilon_{s} \tau^{2}\right)$, where $\beta=1 / k_{\mathrm{B}} T .{ }^{26,27}$ The distance dependent coefficient $C(\tau)$ can be determined using the normalization condition $2 \pi \int_{-1}^{1} d(\cos \theta) P_{\text {ion }}(\tau, \theta)=4 \pi$ which gives ${ }^{27} C(\tau)=\alpha / \sinh \alpha$ with $\alpha \equiv \beta q \mu / \epsilon_{s} \tau^{2}$.

Substituting this $P_{\text {ion }}(\tau, \theta)$ into Eq. (A7), and performing the correction calculation for every water $\left(N_{w}\right.$ of them) in the system, one obtains $(u \equiv \cos \theta)$ 


$$
G=\frac{2 \pi q \mu N_{w}}{\epsilon_{\mathrm{in}} V} \frac{1-\epsilon_{r}}{1+2 \epsilon_{r}} \int_{\tau=R}^{\infty} d \tau \frac{\alpha}{\sinh \alpha} \int_{-1}^{1} d u u e^{-\alpha u}
$$

or, with $\rho_{w} \equiv N_{w} / V$

$$
G=\frac{4 \pi q \mu \rho_{w}}{\epsilon_{\mathrm{in}}}\left(\frac{1-\epsilon_{r}}{1+2 \epsilon_{r}}\right) \int_{\tau=R}^{\infty} d \tau\left(\frac{1}{\alpha}-\frac{\cosh \alpha}{\sinh \alpha}\right) .
$$

For large $\epsilon_{s}$ and $\tau, \alpha$ is small. At this limit, the integrand can be approximated as

$$
\left(\frac{1}{\alpha}-\frac{\cosh \alpha}{\sinh \alpha}\right) \approx-\frac{\alpha}{3}=-\frac{\beta q \mu}{3 \epsilon_{s} \tau^{2}} .
$$

With this approximation, the integral is given as

$$
G=\frac{4 \pi \rho_{w}}{3 \epsilon_{\text {in }}}\left(\frac{\epsilon_{r}-1}{2 \epsilon_{r}+1}\right)\left(\frac{q^{2} \mu^{2}}{k_{\mathrm{B}} T \epsilon_{s}}\right)\left(\frac{1}{R_{c}}\right) .
$$

For $\mu=0.489|e| \AA$ (dipole moment of SPC/E model water), $q=1, \rho_{w}=1 / 30 \AA^{-3}, \epsilon_{\text {in }}=1, \epsilon_{r}=78, T=298 \mathrm{~K}$, and if $G$ and $R_{c}$, respectively, are in the units of $\mathrm{kcal} / \mathrm{mol}$ and Angstroms, Eq. (A12a) can be written as

$$
\Delta G=\frac{39.16}{R_{c}}
$$

${ }^{1}$ C. A. Reynolds, P. M. King, and W. G. Richards, Mol. Phys. 76, 251 (1992).

${ }^{2}$ T. P. Straatsma and J. A. McCammon, Annu. Rev. Phys. Chem. 43, 407 (1992).

${ }^{3}$ T. P. Straatsma and H. J. C. Berendsen, J. Chem. Phys. 89, 5876 (1988).
${ }^{4}$ R. H. Wood, J. Chem. Phys. 103, 6177 (1995).

${ }^{5}$ M. Born, Z. Phys. 1, 45 (1920).

${ }^{6}$ J. G. Kirkwood, J. Chem. Phys. 2, 351 (1934).

${ }^{7}$ H. Alper and R. M. Levy, J. Chem. Phys. 99, 9847 (1993).

${ }^{8}$ L. Wang and J. Hermans, J. Phys. Chem. 99, 12001 (1995).

${ }^{9}$ I. G. Tironi, R. Sperb, P. E. Smith, and W. F. van Gunsteren, J. Chem. Phys. 102, 5451 (1995)

${ }^{10}$ H. Resat and J. A. McCammon, J. Chem. Phys. 104, 7645 (1996).

${ }^{11}$ G. Hummer, L. R. Pratt, and A. E. Garcia, J. Phys. Chem. 100, 1206 (1995).

${ }^{12}$ F. Figueirido, G. S. Del Buono, and R. M. Levy, J. Phys. Chem. B 101, 5622 (1997).

${ }^{13}$ G. Hummer, L. R. Pratt, A. E. Garcia, B. J. Berne, and S. W. Rick, J. Phys. Chem. B 101, 3017 (1997).

${ }^{14}$ H. S. Ashbaugh and R. H. Wood, J. Chem. Phys. 106, 8135 (1997).

${ }^{15}$ M. P. Allen and D. J. Tildesley, Computer Simulation of Liquids (Oxford University, New York, 1987).

${ }^{16}$ (a) H. Resat and M. Mezei, J. Chem. Phys. 99, 6052 (1993); 101, 6126 (1994); (b) T. P. Straatsma and J. A. McCammon, ibid. 95, 1175 (1991).

${ }^{17}$ This equation is the same as Eqs. (5).

${ }^{18}$ H. L. Friedman, Mol. Phys. 29, 1533 (1975).

${ }^{19}$ M. E. Davis, J. Chem. Phys. 100, 5149 (1994).

${ }^{20}$ T. P. Straatsma and J. A. McCammon, J. Comput. Chem. 11, 943 (1990).

${ }^{21}$ K. Soman and T. P. Straatsma (personal communication).

${ }^{22}$ H. J. C. Berendsen, J. R. Grigera, and T. P. Straatsma, J. Phys. Chem. 91, 6269 (1991).

${ }^{23}$ J. P. Ryckaert, G. Ciccotti, and H. J. C. Berendsen, J. Comput. Phys. 23, 327 (1977).

${ }^{24}$ M. E. Davis, J. D. Madura, B. A. Luty, and J. A. McCammon, Comput. Phys. Commun. 62, 187 (1991)

${ }^{25}$ J. D. Jackson, Classical Electrodynamics, 2nd ed. (Wiley, New York, 1975).

${ }^{26}$ H. L. Friedman, A Course in Statistical Mechanics (Prentice-Hall, Englewood Cliffs, New Jersey, 1985).

${ }^{27}$ J.-K. Hyun and T. Ichiye, J. Phys. Chem. B 101, 3596 (1997). 